\title{
Risk factors of locoregional relapse in locally advanced breast cancer treated with neoadjuvant chemotherapy following mastectomy and radiotherapy
}

\author{
Liang Huang ${ }^{1,2, *}$, Sheng Chen ${ }^{1,2, *}$, Wentao T. Yang ${ }^{2,3}$ and Zhiming Shao ${ }^{1,2}$ \\ ${ }^{1}$ Department of Breast Surgery, Fudan University Shanghai Cancer Center/Cancer Institute, Shanghai, China \\ 2 Department of Oncology, Shanghai Medical College, Fudan University, Shanghai, China \\ ${ }^{3}$ Department of Pathology, Fudan University Shanghai Cancer Center/Cancer Institute, Shanghai, China \\ * These authors have contributed equally to this work \\ Correspondence to: Zhiming Shao, email: zhimingshao@yahoo.com \\ Keywords: neoadjuvant chemotherapy, non-pathological complete response, locoregional relapse, annual recurrence rate \\ Received: October 21, $2016 \quad$ Accepted: December 13, $2016 \quad$ Published: December 31, 2016 \\ Copyright: Huang et al. This is an open-access article distributed under the terms of the Creative Commons Attribution License (CC-BY), \\ which permits unrestricted use, distribution, and reproduction in any medium, provided the original author and source are credited.
}

\section{ABSTRACT}

We seek to investigate the prognostic factors that could possibly increase the locoregional recurrence of breast cancer patients who do not achieve pathological complete response after neoadjuvant chemotherapy, and to build a prognostic nomogram to predict patients' outcome. The retrospective analysis included $\mathbf{5 1 0}$ patients who had received neoadjuvant chemotherapy followed by surgery and radiotherapy. 62 locoregional events occurred after a median $\mathbf{6 1}$ months of follow-up. The five-year cumulative incidence of local recurrence and regional recurrence were $8.63 \%$ and $4.31 \%$, respectively. Multivariate analysis revealed that positivity for $\geq$ 4 lymph nodes and Ki-67 index $\geq 14 \%$ were independent factors. According to our prognostic model, the 5-year locoregional free survival rates in the low, intermediate, and high-risk groups were $95.5 \%, 89.1 \%$, and $67.1 \%$, respectively $(p<0.001)$. Annual recurrence curves indicated that the relapse peak after mastectomy emerged in the first 1 year. Positivity for $\geq 4$ lymph nodes and Ki-67 index $\geq 14 \%$ were independent factors for locoregional recurrence. This prognostic model has considerable clinical value in predicting locoregional recurrence, which could help clinicians to design appropriate locoregional treatment specifically and to perform surveillance individually.

\section{INTRODUCTION}

Preoperative or neoadjuvant chemotherapy (NCT) is the standard treatment in locally advanced or inflammatory breast cancer [1]. In patients with operable breast cancer, neoadjuvant chemotherapy can allow increased rate of breast conservation surgery. The use of neoadjuvant chemotherapy has also provided insight into tumour biology and differential responses to treatment. Furthermore, pathologic complete response (pCR) is an early prognostic marker for better long-term outcome [2, $3]$.

When patients receive neoadjuvant chemotherapy, non-pCR status might be a high risk for relapse and metastasis [4]. Optimizing locoregional outcome is important, because these failures can ultimately lead to substantial morbidity, disease progression and death [5, 6]. Receiving NCT was associated with an increased risk of locoregional recurrence (LRR) compared with receiving adjuvant therapy [7]. Retrospective series have demonstrated that the elimination of radiotherapy after NCT in high-risk patients results in an unacceptably high rate of recurrence $[8,9]$. Neoadjuvant chemotherapy followed by definitive surgery consolidated with postmastectomy radiation therapy has become the standard of care for patients with locally advantage breast cancer. It is clear that completion of this therapy is critical to locoregional control, but locoregional failure remains more common among patients with some molecular subtypes [10-12]. 
In contrast, there is limited information on the rates and predictors of LRR for patients who receive neoadjuvant chemotherapy, especially with the use of locoregional external radiotherapy after mastectomy. To address these questions, data from a single cancer institution provided us with the opportunity to examine the rates and patterns of LRR in patients treated with neoadjuvant chemotherapy and to identify independent predictors of LRR in this setting. In this study, we retrospectively analysed 510 neoadjuvant patients with residual tumour who completed mastectomy and postmastectomy radiation therapy (PMRT) in order to identify the independent prognostic factors and create a nomogram to distinguish patients with different outcomes.

\section{PATIENTS AND METHODS}

\section{Study population}

The study cohort in the present study was selected consecutively from patients with locally advanced breast cancer who had received NCT followed by surgery at Shanghai Cancer Centre from 1999 to 2011. The diagnoses were confirmed as invasive carcinoma by core needle biopsy, and node status was assessed by fine needle aspiration of palpable lymph nodes before NCT. After NCT, all patients underwent modified radical mastectomy and were confirmed to be non-pathological complete responders with residual tumour in the breast. Patients who underwent breast-conserving surgery or who did not undergo adjuvant radiotherapy were not eligible for this study. Other exclusion criteria included metastatic disease before surgery, bilateral breast cancer, male breast cancer, and inflammatory breast cancer.

We retrospectively reviewed a series of 510 patients with locally advanced breast cancer who met the above criteria. The NCT regimens included anthracycline-containing, vinorelbine-containing, and taxane-containing regimens for a median of 3 cycles, as previously reported [13]. Due to health insurancerelated limitations, trastuzumab was not utilized before surgery in patients with HER2 overexpression. For all patients, the surgical procedure included mastectomy and axillary lymph node dissection upon completion of NCT. Additional cycles of chemotherapy were subsequently performed to complete a total of 6-8 cycles at the discretion of the treating physician. Radiation was delivered after completion of chemotherapy. The treatment volumes typically included the chest wall and draining lymphatics in the supraclavicular and infraclavicular nodal region (dose prescription was 50 Gy in 25 fractions). The internal nodal region was not routinely irradiated unless pathologically involved. For ER/PR-positive patients, adjuvant endocrine therapy was recommended. Local recurrence was defined as disease recurrence in the ipsilateral breast. Regional recurrence was defined as metastatic disease in the ipsilateral axillary, supraclavicular, infraclavicular or internal mammary lymph nodes. This study is a retrospective study without any type of clinical intervention. The study was conducted according to the principles expressed in the Declaration of Helsinki and approved by the institutional review board of Fudan University Shanghai Cancer Center. All the patients enrolled in this study signed the informed consent voluntarily.

\section{Treatment response}

The clinical responses to neoadjuvant chemotherapy were evaluated based on MRI and ultrasound examinations and in accordance with the Response Evaluation Criteria in Solid Tumors (RECIST) 1.1 [14]. Two experienced pathologists evaluated samples for the presence of a pathological response. The Miller-Payne (MP) grading system was employed to evaluate the decrease in cancer cellularity [15]: No change or some alterations to individual malignant cells without a reduction in overall cellularity was classified as Grade 1; up to a 30\% loss of tumour cells was classified as Grade 2; between an estimated $30 \%$ and $90 \%$ reduction in tumour cells was classified as Grade 3; more than a $90 \%$ loss of tumour cells with only small clusters or widely dispersed individual cells remaining was considered Grade 4; and no invasive malignant cells remaining was considered Grade 5. None of the patients enrolled in this study was considered a Grade 5 responder.

\section{Immunohistochemistry}

Immunohistochemistry (IHC) analysis was performed on paraffin-embedded post-operative tissue sections using standard procedures for breast tumour specimens. The cut-off value for ER positivity and PR positivity was $1 \%$ positive tumour cells with nuclear staining. HER2 was evaluated as $0,1+, 2+$ or $3+$ using circumferential membrane-bound staining; positivity (HER2+) was considered as $3+$ using IHC or with positive florescent in situ hybridization (FISH), whereas cases with 0 to $1+$ or $2+$ without FISH detection were regarded as negative (HER2-). The Ki-67 value was expressed as the percentage of positive cells (at least 1000) with nuclear staining in each case. The following antibodies were used for IHC: ER (M7047, clone 1D5, Dako, Produktionsvej, Glostrup, Denmark), PR (M3569, clone PR 636, Dako), HER2 (A0485, polyclonal rabbit antibody, Dako), and Ki67 (MIB-1, Dako). 
Table 1: Patients characteristics at baseline and clinical evaluation

\begin{tabular}{|l|c|c|c|c|c|}
\hline & $\begin{array}{c}\text { Luminal A } \\
\mathbf{N = \mathbf { 2 0 2 }}\end{array}$ & $\begin{array}{c}\text { Luminal B } \\
\mathbf{N}=\mathbf{1 0 5}\end{array}$ & $\begin{array}{c}\text { HER2+ } \\
\mathbf{N}=\mathbf{6 2}\end{array}$ & $\begin{array}{c}\text { TNBC } \\
\boldsymbol{N}=\mathbf{1 4 1}\end{array}$ & $\boldsymbol{P}$ value \\
\hline Age & & & & & 0.232 \\
\hline$\geq 50 \mathrm{y}$ & 97 & 52 & 36 & 60 & \\
\hline$<50 \mathrm{y}$ & 105 & 53 & 26 & 81 & \\
\hline Menopause status & & & & & 0.719 \\
\hline premenopause & 115 & 61 & 31 & 82 & \\
\hline postmenopause & 87 & 44 & 31 & 59 & \\
\hline cT stage & & & & & 0.322 \\
\hline T2 & 66 & 35 & 15 & 47 & \\
\hline T3 & 98 & 56 & 35 & 79 & \\
\hline T4 & 38 & 14 & 12 & 15 & \\
\hline clinical N status & & & & & 0.478 \\
\hline positive & 143 & 80 & 48 & 109 & \\
\hline negative & 59 & 25 & 14 & 32 & \\
\hline chemotherapy regimen & & & & & 0.075 \\
\hline non-Taxel & 129 & 68 & 42 & 108 & \\
\hline Taxel & 73 & 37 & 20 & 33 & \\
\hline clinical response & 131 & 63 & 37 & 78 & \\
\hline CR/PR & 71 & 42 & 25 & 63 & \\
\hline SD/PD & & & & & 0.360 \\
\hline
\end{tabular}

\section{Literature search and literature-based data extraction}

We searched the PubMed, Web of Science, and MEDLINE databases (updated to May 1, 2015) using the following search terms: "annual" and "recurrence" and "breast cancer." Eligible studies and their references were retrieved and examined carefully. The literature inclusion criteria were as follows: (a) evaluation of ARR after surgery for primary breast cancer, (b) availability of information for ARR (numerical data or graphic data), and (c) full text published in English. Relevant information was carefully extracted from all eligible publications.

\section{Statistical analysis}

Locoregional relapse-free survival (LRFS, defined as disease recurrence in the chest wall or regional lymph nodes) and distant relapse-free survival (DRFS, defined as distant disease metastasis) were calculated from the date of surgery to the date of disease relapse. Overall survival (OS) was calculated from the date of surgery to the date of death or last follow-up. Patients without relapse events or death were censored at the last follow-up. Multivariate analyses were performed with the Cox proportional model to determine the effects of independent prognostic factors. Survival curves were estimated using the Kaplan-Meier method, and the log-rank test was used to determine significance. All P-values reported are two-sided and were calculated at a significance level of 0.05 . All statistical procedures were performed with SPSS 13.0 and STATA 11.0.

\section{RESULTS}

We analyzed 510 breast cancer patients treated by neoadjuvant chemotherapy and underwent modified radical mastectomy followed by radiotherapy in Shanghai Cancer Centre between 1999 to 2011. These patients were divided by 4 groups based on the 2011 St. Gallen guidelines [16]. Table 1 lists the clinicopathological characteristics by constructed subtype. The median age of all patients was 49 years old (range 24-75 years). $74.5 \%$ of patients had clinically positive lymph nodes, and $43.3 \%$ of patients were in menopause. $96.1 \%$ of patients were diagnosed with invasive ductal carcinoma. Patients received two main regimens, including taxolbased regimens and non-taxol based regimens. There were no significant differences in the distribution of neoadjuvant regimens between the subtypes. The other clinicopathological features of patients were wellbalanced. Overall, $60.6 \%$ (95\% CI: $56.3 \%-64.9 \%$ ) of the patients experienced a clinical objective response (CR or PR) as assessed by palpation, ultrasonography and/or MRI.

After a median follow-up of 61 months (range 5-128 months), there were 40 isolated local recurrences, 17 isolated regional recurrences and 5 locoregional recurrences. Sixteen patients $(25.8 \%)$ occurred 
Table 2: Distribution of local-regional recurrences and metastasis by constructed molecular subtype

\begin{tabular}{|l|c|c|c|c|c|c|}
\hline & overall & Luminal A & Luminal B & HER2+ & TNBC & $P$ value \\
\hline Local & 45 & 9 & 15 & 7 & 14 & 0.025 \\
\hline Reginal & 22 & 7 & 10 & 2 & 3 & 0.028 \\
\hline LRR & 62 & 13 & 24 & 9 & 16 & $<0.001$ \\
\hline Metastasis & 145 & 35 & 43 & 18 & 49 & $<0.001$ \\
\hline Cohort & 510 & 202 & 105 & 62 & 141 & \\
\hline
\end{tabular}

Table 3: Univariate and multivariate analysis of time to locoregional recurrence

\begin{tabular}{|c|c|c|c|c|c|c|}
\hline & \multicolumn{3}{|c|}{ Univariate } & \multicolumn{3}{|c|}{ Multivariate } \\
\hline & HR & $95 \% \mathrm{CI}$ & $P$ & HR & $95 \% \mathrm{CI}$ & $P$ \\
\hline Age $(\geq 50 \mathrm{y} v s .<50 \mathrm{y})$ & 1.387 & $0.841-2.288$ & 0.200 & & - & \\
\hline Menopause status (pre $v s$. post) & 1.493 & $0.907-2.459$ & 0.115 & & - & \\
\hline $\mathrm{pT}(\mathrm{T} 1 v s . \mathrm{T} 2 / 3)$ & 2.158 & $1.207-3.860$ & 0.010 & 1.625 & $0.902-2.926$ & 0.106 \\
\hline $\mathrm{pN}(\mathrm{N} 0 / 1$ vs. $\mathrm{N} 2 / 3)$ & 3.489 & $1.950-6.242$ & $<0.001$ & 3.295 & $1.828-5.939$ & $<0.001$ \\
\hline MP (3/4 vs. 2-0) & 1.594 & $0.968-2.623$ & 0.067 & & - & \\
\hline ER (positive $v s$. negative) & 0.724 & $0.440-1.193$ & 0.205 & & - & \\
\hline PR (positive $v s$. negative) & 0.806 & $0.488-1.333$ & 0.401 & & - & \\
\hline HER2 (positive $v s$. negative) & 1.617 & $0.942-2.776$ & 0.081 & & - & \\
\hline Ki67 $(\geq 14 \%$ vs. $<14 \%)$ & 3.105 & $1.884-5.119$ & $<0.001$ & 2.897 & $1.701-4.937$ & $<0.001$ \\
\hline nuclear grade $(1 / 2$ vs. 3$)$ & 2.205 & $1.310-3.710$ & 0.003 & 1.371 & $0.787-2.390$ & 0.266 \\
\hline LVI (positive $v s$. negative) & 0.936 & $0.545-1.606$ & 0.809 & & - & \\
\hline clinical response $(\mathrm{CR} / \mathrm{PR} v s . \mathrm{SD} / \mathrm{PD})$ & 1.506 & $0.915-2.479$ & 0.107 & & - & \\
\hline Chemo regimen (non-Taxel vs Taxel) & 0.946 & $0.551-1.623$ & 0.839 & & - & \\
\hline
\end{tabular}

synchronously with distant metastasis, and no LRR was detected after distant failure. Fifteen patients had recurrences in the supraclavicular field. The 5-year cumulative LRR-free survival rate was $88.0 \%$ (95\% CI: $85.2 \%-90.1 \%)$. The 5-year overall survival and diseasefree survival rates were $79.0 \%(75.5 \%-82.6 \%)$ and $63.1 \%$ (58.9\%-67.3\%), respectively. The luminal A subtype had the lowest local-region recurrence rate $(6.44 \%)$, and the luminal B subtype had the highest LRR rate $(22.86 \%)$. The distribution of local and regional recurrences by different molecular subtype was shown in Table 2. The cumulative incidence of local and regional recurrence was significantly greater in patients with the luminal B subtype $(p<0.001)$. The hormone receptor positive and HER2-negative subgroups had similar local and regional recurrences rate, whereas the other subgroups had a higher rate of local failure $(p=0.012)$.

In OS analysis, patients with local recurrence had a worse outcome (HR: 4.255, 95\% CI: 2.713-6.672, $P$ $<0.001$ ), whereas regional recurrence trended toward a significant difference (HR: 1.974, 95\% CI: 0.961-4.053, $P$ $=0.064)$. Among patients with local-region failure, HER2positive and TNBC patients had a shorter survival time than those with luminal types (HR: 2.356, 95\% CI: $1.157-$ 4.798, $P=0.018$ ). Pathological tumour stage, pathological nodal status, Ki67 index and nuclear grade were all significantly associated with LRR-free survival rate in univariate analysis (Table 3). However, in multivariate analysis of the four covariates, pathological nodal status and ki67 index still had a significant impact on LRR-free survival.

After radiotherapy, $48.4 \%$ of locoregional recurrences occurred within the 1 st year, 19.4\% occurred within the $2^{\text {nd }}$ year, $11.3 \%$ occurred within the $3^{\text {rd }}$ year, and the remaining $21.0 \%$ of LRR occurred after 3 years. The prognostic model was established based on the sum of both independent prognostic factors, with positivity for $\geq 4$ lymph nodes and Ki-67 index $\geq 14 \%$ individually contributing 1 point to the risk score. The patients were assigned to a low-risk group (0 point), median-risk group (1 point) and high-risk group ( 2 points). These subgroups had significantly different outcomes (Figure 1A, $P<$ 0.0001 for LRFS; figure $1 \mathrm{~B}, P<0.0001$ for OS).

The aim of the present study was to show the annual LRR rate among these 3 groups using our singleinstitution data, as well as to review relevant literaturebased data to compare with our observations (Figure 2). Four publications that reported ARR (annual recurrence rate) after surgery for primary breast cancer were identified as eligible [17-20]. In the relevant literature, the annual risk of local-regional recurrence peaked between two and three years after the initial diagnosis. In contrast, the ARR curve of the high risk subgroup for neoadjuvant patients exhibited one peak near 1 year (17\% per annum). The 
median and low-risk subgroups did not have an obvious recurrence peak.

\section{DISCUSSION}

We described the rates and patterns of LRR in non-pathological complete responders who received neoadjuvant chemotherapy. In our study focusing on non-pCR patients, we excluded the pCR patients, who had the best locoregional control. Overall, patients with more advanced nodal disease and a high Ki67 index had a higher risk of loco-regional relapse. The existence of loco-

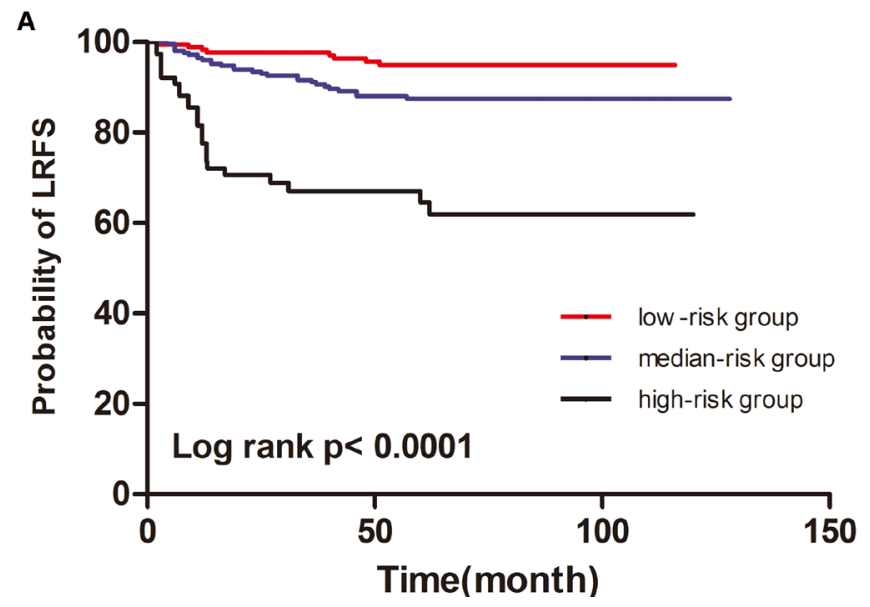

regional recurrence was associated with a worse overall survival, and efforts to improve axillary control might prove of benefit in this population [5]. It remains unclear why LRR occurs after local therapy such as mastectomy and adjuvant radiation, but the results might be explained by the self-seeding hypothesis, in which it is postulated that residual cells in the breast might act as a pool of potential metastases and could seed distant sites as well as the primary tumour itself [21].

Recent publications have revealed locoregional control rates from $80 \%$ to $90 \%$ in the neoadjuvant setting, which is similar to the observation in our study [2224]. The importance of the underlying biology of breast

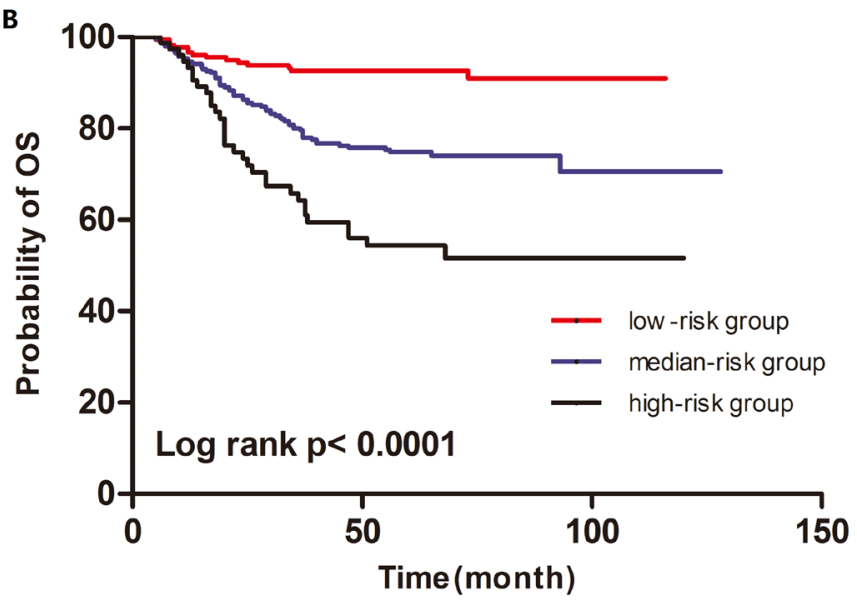

Figure 1: Kaplan-Meier curves for LRFS (A) and OS (B) of according to the prognostic model

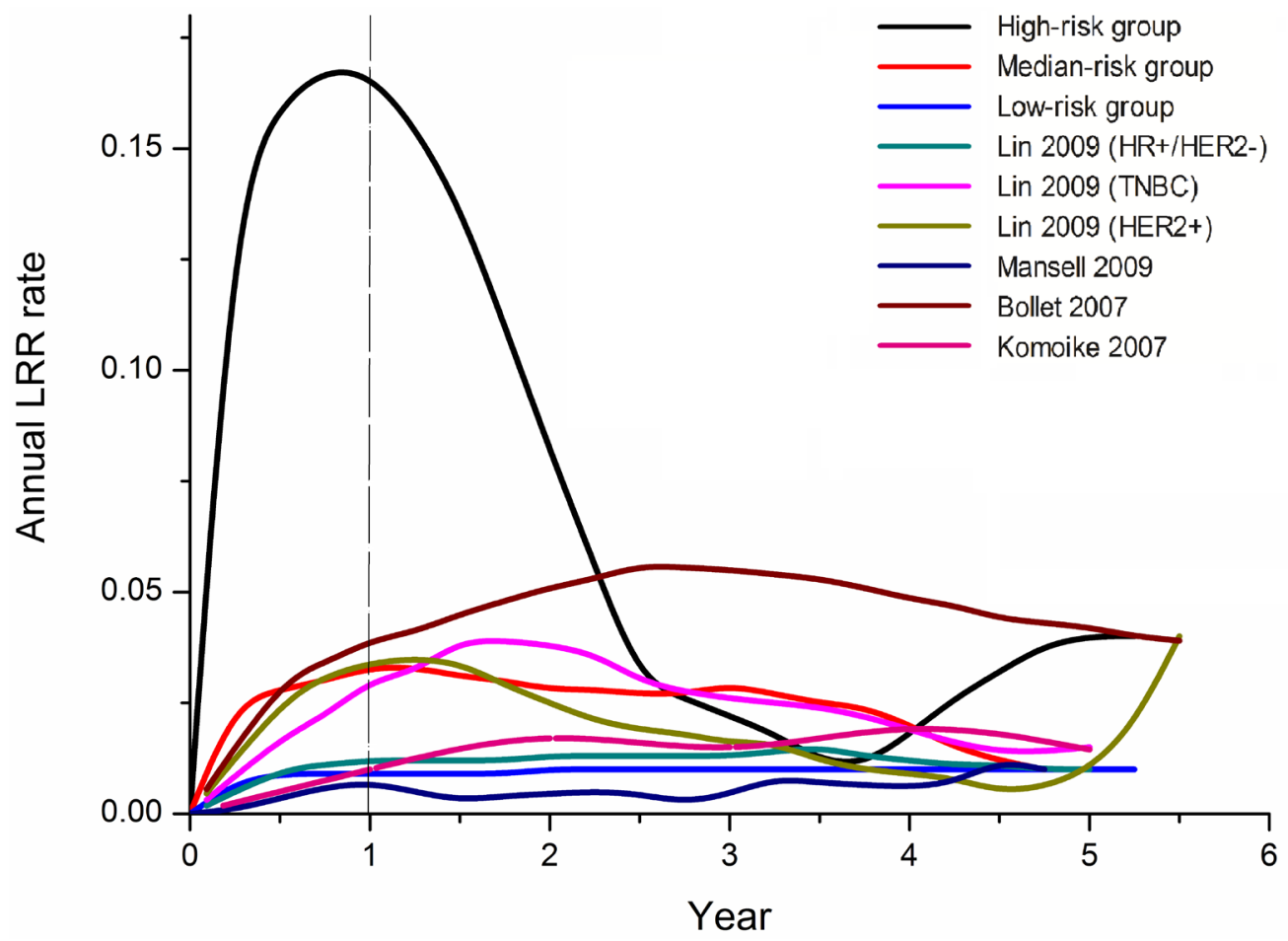

Figure 2: Annual recurrence rate curves derived from our data and relevant studies 
tumours in predicting outcome has been demonstrated by microarray analyses that identified molecular subtypes. Clinicians have used hormone receptor, HER2 status and the Ki67 index to group tumours into constructed subtypes. Patients in the luminal A subtype had the lowest LRR rate, whereas those in the luminal B subtype had the highest LRR rate. Without neoadjuvant treatment, the LRR rate after mastectomy was only $8 \%$ for luminal A tumours compared with $22 \%$ for luminal B tumours [25]. There was no clear association between the risk of loco-regional recurrence and MP stage, HR/HER2 status. Some type of breast-conserving surgical intervention is likely to be warranted, regardless of whether neoadjuvant or adjuvant treatment is adopted and regardless of the patient's initial clinical response. The local and regional recurrence rates were similar in the $\mathrm{HR}+$ subgroup, whereas the aggressive subtypes such as HER2+ positive and TNBC had higher local failure rates. There is not sufficient evidence supporting this result in other reports [26]. Patients with aggressive subtypes with LRR have a shorter overall survival than those with luminal subtypes.

Although locoregional failures occurred rarely for the entire cohort, patients with involvement of more than 4 lymph nodes remained at increased risk of LRR $(p<0.001)$. The pathological status of lymph nodes was significantly related a worse relapse rate and metastasis in both the neoadjuvant and adjuvant settings [24, 25, 27]. Even after a 10 -year follow-up, LRR is $16 \%$ for patients with four or more positive nodes [8]. Comprehensive nodal irradiation therapy to the full axilla might be of benefit for patients with a greater burden of residual positive disease in the axilla ( $\geq 4$ positive nodes) [6]. However, for patients with one to three positive lymph nodes after chemotherapy, radiation did not yield a locoregional control benefit [8]. There was a very low LRR rate $(1.9 \%)$ in patients who had pathologically negative lymph nodes after NCT and radiotherapy [11].

The proliferation marker Ki67 has been suggested as a promising cancer biomarker. Despite the high number of significant biomarker studies for Ki67, this marker was still used with reluctance in the clinical setting. To describe biologically the proliferative capacity of residual disease, Ki67 on the residual tumour was analysed. A total of 1150 patients from the GeparTrio study were divided into 4 groups based on different Ki67 levels, which were significantly related to DFS and OS [28]. The Ki67 cut point used in different studies varies between 5\% and $34 \%$ [29, 30]. Our cut-off value is based on the $2013 \mathrm{St}$. Gallen Consensus [31]. Compared with pre-treatment measurements or changes from before and after treatment, post-treatment Ki67 was more relevant [32]. Furthermore, some research has added that post-treatment Ki67 improves the prediction of long-term outcome [33].

The rate of local recurrences was in line with those reported in the literature, which range from $12 \%$ to $30 \%$, mostly dependent on the length of follow-up. In the adjuvant cohort, the first recurrence peak after mastectomy occurred at 2 years [34]. The annual risk of locoregional recurrence has an early peak during the first year following treatment in the neoadjuvant setting [19]. In the high-risk group, the incremental gains of locoregional control are theoretically greater. These high-risk patients might benefit most from dose-dense chemotherapy and comprehensive irradiation of the full axilla as a component of PMRT. On the other hand, novel compounds are in preparation to be assessed in the post-neoadjuvant setting for non-pCR patients. In the PENELOPE study, a novel cyclin-D kinase 4/6 inhibitor is being explored in addition to endocrine treatment. The Katherine study focuses on TDM1, which will be randomly compared with the continuation of trastuzumab. In CREATE-X trial, adjuvant capecitabine improved outcomes in women with HER2negative breast cancer who have residual invasive disease after neoadjuvant chemotherapy. However, in the lowrisk group, the annual hazard ratio was less than $0.3 \%$. Therefore, these patients are candidates to further reduce the extent of locoregional treatment.

Our study has limitations because of its retrospective character. Furthermore, patients received different chemotherapy regimens and HER2 positive patients did not receive trastuzumab as part of the neoadjuvant treatment. Another limitation of our study is the small number of patients from which to draw meaningful conclusions about patients with luminal B/HER2- and HER2+. The appropriate identification of patients at high risk of relapse following neoadjuvant chemotherapy could enable the characterization of drivers of drug resistance [35]. High throughput technology with DNA, RNA or proteins is now available to look for a breast cancer prognostic profile. However, our nomogram could be a useful tool for predicting the risk of LRR in non-pCR responders. This would be of the utmost importance in the selection of patients who would benefit from standard treatment and those who would not. However, this nomogram requires validation in other independent data sets. Furthermore, it highlights the significance of optimizing outcomes with local and regional adjuvant therapies. The nomogram has important implications for the risk of LRR and might help to identify patients who might benefit from novel strategies to improve locoregional control. The potential is also highlighted for the design of post-neoadjuvant adjuvant studies in these high-risk populations.

\section{ACKNOWLEDGMENTS AND GRANT SUPPORT}

This research was supported by the National Natural Science Foundation of China (81302298 and 81502289). The funders had no role in the study design, data collection and analysis, decision to publish, or preparation of the manuscript. 


\section{CONFLICTS OF INTERESTS}

The authors declare no competing financial interests.

\section{Author contributions}

L Huang, S Chen and ZM Shao conceived and designed the study. L Huang sorted and analyzed the clinicopathological data. S Chen and WT Yang prepared the figures and tables and wrote the main manuscript. All of the authors reviewed the manuscript.

\section{REFERENCES}

1. Chia S, Swain SM, Byrd DR, Mankoff DA. Locally advanced and inflammatory breast cancer. J Clin Oncol. 2008; 26: 786-90. doi: 10.1200/JCO.2008.15.0243 26/5/786.

2. Kong X, Moran MS, Zhang N, Haffty B, Yang Q. Metaanalysis confirms achieving pathological complete response after neoadjuvant chemotherapy predicts favourable prognosis for breast cancer patients. Eur J Cancer. 2011; 47: 2084-90. doi: S0959-8049(11)00418-7 [pii] 10.1016/j. ejca.2011.06.014.

3. Prowell TM, Pazdur R. Pathological complete response and accelerated drug approval in early breast cancer. N Engl J Med. 2012; 366: 2438-41. doi: 10.1056/NEJMp1205737.

4. Rastogi P, Anderson SJ, Bear HD, Geyer CE, Kahlenberg MS, Robidoux A, Margolese RG, Hoehn JL, Vogel VG, Dakhil SR, Tamkus D, King KM, Pajon ER, et al. Preoperative chemotherapy: updates of National Surgical Adjuvant Breast and Bowel Project Protocols B-18 and B-27. J Clin Oncol. 2008; 26: 778-85. doi: 26/5/778 [pii] 10.1200/JCO.2007.15.0235.

5. Clarke M, Collins R, Darby S, Davies C, Elphinstone P, Evans E, Godwin J, Gray R, Hicks C, James S, MacKinnon E, McGale P, McHugh T, et al. Effects of radiotherapy and of differences in the extent of surgery for early breast cancer on local recurrence and 15-year survival: an overview of the randomised trials. Lancet. 2005; 366: 2087-106. doi: S01406736(05)67887-7 [pii] 10.1016/S0140-6736(05)67887-7.

6. Saigal K, Hurley J, Takita C, Reis IM, Zhao W, Rodgers SE, Wright JL. Risk factors for locoregional failure in patients with inflammatory breast cancer treated with trimodality therapy. Clin Breast Cancer. 2013; 13: 335-43. doi: 10.1016/j.clbc.2013.04.002 S1526-8209(13)00106-7.

7. Mauri D, Pavlidis N, Ioannidis JP. Neoadjuvant versus adjuvant systemic treatment in breast cancer: a metaanalysis. J Natl Cancer Inst. 2005; 97: 188-94. doi: 10.1093/ jnci/dji021.

8. Huang EH, Tucker SL, Strom EA, McNeese MD, Kuerer HM, Buzdar AU, Valero V, Perkins GH, Schechter NR, Hunt KK, Sahin AA, Hortobagyi GN, Buchholz TA. Postmastectomy radiation improves local-regional control and survival for selected patients with locally advanced breast cancer treated with neoadjuvant chemotherapy and mastectomy. J Clin Oncol. 2004; 22: 4691-9. doi: 22/23/4639 [pii] 10.1200/JCO.2004.11.129.

9. McGuire SE, Gonzalez-Angulo AM, Huang EH, Tucker SL, Kau SW, Yu TK, Strom EA, Oh JL, Woodward WA, Tereffe W, Hunt KK, Kuerer HM, Sahin AA, et al. Postmastectomy radiation improves the outcome of patients with locally advanced breast cancer who achieve a pathologic complete response to neoadjuvant chemotherapy. Int J Radiat Oncol Biol Phys. 2007; 68: 1004-9. doi: S03603016(07)00139-3 [pii] 10.1016/j.ijrobp.2007.01.023.

10. Arsenault D, Hurley J, Takita C, Reis IM, Zhao W, Rodgers S, Wright JL. Predictors of Locoregional Outcome in HER2-Overexpressing Breast Cancer Treated With Neoadjuvant Chemotherapy. Am J Clin Oncol. 2013. doi: 10.1097/COC.0b013e31829d1eb8.

11. Wright JL, Takita C, Reis IM, Zhao W, Saigal K, Wolfson A, Markoe A, Moller M, Hurley J. Predictors of locoregional outcome in patients receiving neoadjuvant therapy and postmastectomy radiation. Cancer. 2013; 119: 16-25. doi: 10.1002/cncr.27717.

12. Haffty BG, McCall LM, Ballman KV, McLaughlin S, Jagsi R, Ollila DW, Hunt KK, Buchholz TA, Boughey JC. Patterns of Local-Regional Management Following Neoadjuvant Chemotherapy in Breast Cancer: Results From ACOSOG Z1071 (Alliance). Int J Radiat Oncol Biol Phys. 2016; 94: 493-502. doi: 10.1016/j.ijrobp.2015.11.005.

13. Chen S, Chen CM, Yu KD, Yang WT, Shao ZM. A prognostic model to predict outcome of patients failing to achieve pathological complete response after anthracyclinecontaining neoadjuvant chemotherapy for breast cancer. J Surg Oncol. 2012; 105: 577-85. doi: 10.1002/jso.22140.

14. Eisenhauer EA, Therasse P, Bogaerts J, Schwartz LH, Sargent D, Ford R, Dancey J, Arbuck S, Gwyther S, Mooney M, Rubinstein L, Shankar L, Dodd L, et al. New response evaluation criteria in solid tumours: revised RECIST guideline (version 1.1). Eur J Cancer. 2009; 45: 228-47. doi: 10.1016/j.ejca.2008.10.026.

15. Ogston KN, Miller ID, Payne S, Hutcheon AW, Sarkar TK, Smith I, Schofield A, Heys SD. A new histological grading system to assess response of breast cancers to primary chemotherapy: prognostic significance and survival. Breast. 2003; 12: 320-7.

16. Goldhirsch A, Wood WC, Coates AS, Gelber RD, Thurlimann B, Senn HJ, Panel M. Strategies for subtypes-dealing with the diversity of breast cancer: highlights of the St. Gallen International Expert Consensus on the Primary Therapy of Early Breast Cancer 2011. Ann Oncol. 2011; 22: 1736-47. doi: 10.1093/annonc/mdr304.

17. Lin Y, Yin W, Yan T, Zhou L, Di G, Wu J, Shen Z, Shao $\mathrm{Z}$, Lu J. Site-specific relapse pattern of the triple negative tumors in Chinese breast cancer patients. BMC Cancer. 2009; 9: 342. doi: 10.1186/1471-2407-9-342.

18. Mansell J, Monypenny IJ, Skene AI, Abram P, Carpenter 
R, Gattuso JM, Wilson CR, Angerson WJ, Doughty JC. Patterns and predictors of early recurrence in postmenopausal women with estrogen receptor-positive early breast cancer. Breast Cancer Res Treat. 2009; 117: 91-8. doi: 10.1007/s10549-008-0291-z.

19. Bollet MA, Sigal-Zafrani B, Mazeau V, Savignoni A, de la Rochefordiere A, Vincent-Salomon A, Salmon R, Campana F, Kirova YM, Dendale R, Fourquet A. Age remains the first prognostic factor for loco-regional breast cancer recurrence in young ( $<40$ years) women treated with breast conserving surgery first. Radiother Oncol. 2007; 82: 272-80. doi: S0167-8140(07)00016-3 [pii] 10.1016/j. radonc.2007.01.001.

20. Komoike Y, Akiyama F, Iino Y, Ikeda T, Akashi-Tanaka S, Ohsumi S, Kusama M, Sano M, Shin E, Suemasu K, Sonoo H, Taguchi T, Nishi T, et al. Ipsilateral breast tumor recurrence (IBTR) after breast-conserving treatment for early breast cancer: risk factors and impact on distant metastases. Cancer. 2006; 106: 35-41. doi: 10.1002/ cncr.21551.

21. Comen E, Norton L, Massague J. Clinical implications of cancer self-seeding. Nat Rev Clin Oncol. 2011; 8: 369-77. doi: 10.1038/nrclinonc.2011.64.

22. Nagar H, Mittendorf EA, Strom EA, Perkins GH, Oh JL, Tereffe W, Woodward WA, Gonzalez-Angulo AM, Hunt KK, Buchholz TA, Yu TK. Local-regional recurrence with and without radiation therapy after neoadjuvant chemotherapy and mastectomy for clinically staged T3N0 breast cancer. Int J Radiat Oncol Biol Phys. 2011; 81: 782 7. doi: 10.1016/j.ijrobp.2010.06.027.

23. Mamounas EP, Anderson SJ, Dignam JJ, Bear HD, Julian TB, Geyer CE Jr, Taghian A, Wickerham DL, Wolmark N. Predictors of locoregional recurrence after neoadjuvant chemotherapy: results from combined analysis of National Surgical Adjuvant Breast and Bowel Project B-18 and B-27. J Clin Oncol. 2012; 30: 3960-6. doi: 10.1200/ JCO.2011.40.8369.

24. Wu SG, Li Q, Zhou J, Sun JY, Li FY, Lin Q, Lin HX, Gaun XX, He ZY. Using the Lymph Node Ratio to Evaluate the Prognosis of Stage II/III Breast Cancer Patients Who Received Neoadjuvant Chemotherapy and Mastectomy. Cancer Res Treat. 2014. doi: 10.4143/crt.2014.039.

25. Voduc KD, Cheang MC, Tyldesley S, Gelmon K, Nielsen TO, Kennecke H. Breast cancer subtypes and the risk of local and regional relapse. J Clin Oncol. 2010; 28: 1684-91. doi: 10.1200/JCO.2009.24.9284.

26. Caudle AS, Yu TK, Tucker SL, Bedrosian I, Litton JK, Gonzalez-Angulo AM, Hoffman K, Meric-Bernstam F, Hunt KK, Buchholz TA, Mittendorf EA. Local-regional control according to surrogate markers of breast cancer subtypes and response to neoadjuvant chemotherapy in breast cancer patients undergoing breast conserving therapy. Breast Cancer Res. 2012; 14: R83. doi: 10.1186/bcr3198.

27. Cheng SH, Horng CF, Clarke JL, Tsou MH, Tsai SY, Chen CM, Jian JJ, Liu MC, West M, Huang AT, Prosnitz LR.
Prognostic index score and clinical prediction model of local regional recurrence after mastectomy in breast cancer patients. Int J Radiat Oncol Biol Phys. 2006; 64: 1401-9. doi: 10.1016/j.ijrobp.2005.11.015.

28. Denkert C, Loibl S, Muller BM, Eidtmann H, Schmitt WD, Eiermann W, Gerber B, Tesch H, Hilfrich J, Huober J, Fehm T, Barinoff J, Jackisch C, et al. Ki67 levels as predictive and prognostic parameters in pretherapeutic breast cancer core biopsies: a translational investigation in the neoadjuvant GeparTrio trial. Ann Oncol. 2013; 24: 2786-93. doi: 10.1093/annonc/mdt350 mdt350 [pii].

29. Burcombe R, Wilson GD, Dowsett M, Khan I, Richman PI, Daley F, Detre S, Makris A. Evaluation of Ki-67 proliferation and apoptotic index before, during and after neoadjuvant chemotherapy for primary breast cancer. Breast Cancer Res. 2006; 8: R31. doi: bcr1508 [pii] 10.1186/ bcr1508.

30. Goldhirsch A, Ingle JN, Gelber RD, Coates AS, Thurlimann B, Senn HJ. Thresholds for therapies: highlights of the St Gallen International Expert Consensus on the primary therapy of early breast cancer 2009. Ann Oncol. 2009; 20: 1319-29. doi: 10.1093/annonc/mdp322 mdp322.

31. Goldhirsch A, Winer EP, Coates AS, Gelber RD, PiccartGebhart M, Thurlimann B, Senn HJ. Personalizing the treatment of women with early breast cancer: highlights of the St Gallen International Expert Consensus on the Primary Therapy of Early Breast Cancer 2013. Ann Oncol. 2013; 24: 2206-23. doi: 10.1093/annonc/mdt303 mdt303.

32. von Minckwitz G. Neoadjuvant therapy: what are the lessons so far? Hematol Oncol Clin North Am. 2013; 27 : 767-84. doi: 10.1016/j.hoc.2013.05.006.

33. Sheri A, Smith IE, Johnston SR, A'Hern R, Nerurkar A, Jones RL, Hills M, Detre S, Pinder SE, Symmans WF, Dowsett M. Residual proliferative cancer burden to predict long-term outcome following neoadjuvant chemotherapy. Ann Oncol. 2015; 26: 75-80. doi: 10.1093/annonc/mdu508.

34. Yu KD, Li S, Shao ZM. Different annual recurrence pattern between lumpectomy and mastectomy: implication for breast cancer surveillance after breast-conserving surgery. Oncologist. 2011; 16: 1101-10. doi: 10.1634/ theoncologist.2010-0366.

35. Balko JM, Cook RS, Vaught DB, Kuba MG, Miller TW, Bhola NE, Sanders ME, Granja-Ingram NM, Smith JJ, Meszoely IM, Salter J, Dowsett M, Stemke-Hale K, et al. Profiling of residual breast cancers after neoadjuvant chemotherapy identifies DUSP4 deficiency as a mechanism of drug resistance. Nat Med. 2012; 18: 1052-9. doi: 10.1038/nm.2795. 